\title{
Magnetic excitations in vanadium spinels
}

\author{
N. B. Perkins ${ }^{1,2}$ and O. Sikora ${ }^{3}$ \\ ${ }^{1}$ Institute fur Theoretische Physik, TU Braunschweig, Mendelssohnstrasse 3, 38106 Braunschweig, Germany \\ ${ }^{2}$ Bogoliubov Laboratory of Theoretical Physics, JINR, Dubna, Russia \\ ${ }^{3}$ MPIPKS, Nöthnitzer Str. 38, 01187 Dresden, Germany
}

(Dated: September 11, 2021)

\begin{abstract}
We study magnetic excitations in vanadium spinel oxides $\mathrm{AV}_{2} \mathrm{O}_{4}(\mathrm{~A}=\mathrm{Zn}, \mathrm{Mg}, \mathrm{Cd})$ using two models: first one is a superexchange model for vanadium $S=1$ spins, second one includes in addition spin-orbit coupling, and crystal anisotropy. We show that the experimentally observed magnetic ordering can be obtained in both models, however the orbital ordering is different with and without spin-orbit coupling and crystal anisotropy. We demonstrate that this difference strongly affects the spin-wave excitation spectrum above the magnetically ordered state, and argue that the neutron measurement of such dispersion is a way to distinguish between the two possible orbital orderings in $\mathrm{AV}_{2} \mathrm{O}_{4}$.
\end{abstract}

PACS numbers: 71.27.+a, 75.30.Mb, 75.20.Hr, 75.10.-b

\section{INTRODUCTION}

Due to geometrical frustration, transition metal spinel oxides with a general formula $A B_{2} \mathrm{O}_{4}$ display a variety of unusual low-temperature properties. The spin dynamics of these systems is usually described by a Heisenberg antiferromagnet on the pyrochlore lattice. This model is rather peculiar and its classical ground state is highly degenerate. Exact degeneracy can be lifted by various mechanisms, but the system still possesses many competing spin configurations with almost equal energies. As a result, when the temperature goes down, the system can evolve in a variety of ways: it can remain spin liquid down to the lowest temperatures due to quantum fluctuations, or choose a particular configuration either via the strongest order from disorder mechanism or through a structural phase transition which lowers the local symmetry of the lattice.

A peculiarity of transition metal spinels is that their magnetic ions often possess also an orbital degree of freedom. This extra degree of freedom modulates the spin exchange and can at least partially lift the geometrical degeneracy of the underlying lattice. However, the orbital degrees of freedom in geometrically frustrated lattices are by themselves frustrated, i.e., many different orbital configurations have the same energy. In this situation, the ordering in the orbital sector is coupled to the ordering in the spin sector, and the selection of a true ground state configuration becomes a non-trivial phenomena.

Of particular interest is the orbital ordering in the $t_{2 g}$ systems on the pyrochlore lattice. In this work we study vanadium spinels of the type $A \mathrm{~V}_{2} \mathrm{O}_{4}$, where $A$ is a divalent ion like $\mathrm{Cd}^{2+}, \mathrm{Zn}^{2+}$, or $\mathrm{Mg}^{2+}$. In these compounds, magnetically active $\mathrm{V}^{3+}$ ions form a pyrochlore lattice and have two $3 d$ electrons in $t_{2 g}$-orbitals. Due to strong Hund's interaction, these two electrons form a state with $S=1$. All $A \mathrm{~V}_{2} \mathrm{O}_{4}$ compounds show qualitatively similar structural and magnetic behavior, independently of what the divalent $\mathrm{A}$ ion is, and undergo two phase transitions - a structural one and an antiferromagnetic one. We will be mainly discussing the physics of $\mathrm{ZnV}_{2} \mathrm{O}_{4}$. The structural transition occurs at a temperature $T_{S} \sim 50 \mathrm{~K} \cdot 1$ Below $T_{S}$, the lattice shows a tetragonal dis- tortion - the vanadium octahedra $\mathrm{VO}_{6}$ are uniformly flattened along the $c$ axis, and the symmetry is lowered from the cubic one to $I 4_{1} / a m d$, which is the highest-symmetry tetragonal space group for the spinel structure. The antiferromagnetic (AFM) transition occurs at a slightly lower temperature $T_{N}$ of about $40 \mathrm{~K} \cdot \frac{2,3}{.3}$ This temperature is significantly lower than the Curie-Weiss temperature $T_{C W} \sim 1000 \mathrm{~K}$, extracted from high temperature susceptibility, $\frac{4}{ \pm}$ which underlines the importance of geometrical frustration.

The magnetic structure of $\mathrm{AV}_{2} \mathrm{O}_{4}$ spinels at $T<T_{N}$ was first proposed by Niziol ${ }^{2}$ and recently confirmed by Reehius et al. in Ref. 3. Along the diagonal $[110] /[\overline{1} 10]$ direction in the $x y$-plane, the ordering is antiferromagnetic $+-+\ldots$, while along the two other diagonal directions $[011] /[0 \overline{1} 1]$ in the $y z$ plane and $[101] /[\overline{1} 01]$ in the $x z$-plane, the spin ordering is in the form $++-\ldots++-\ldots$ (see Fig. 1).

At high temperatures $T>T_{S}>T_{N}$, inelastic neutron scattering data by Lee $e t$ al. $\frac{5}{.}$ on the powder sample of $\mathrm{ZnV}_{2} \mathrm{O}_{4}$ still show strong low energy magnetic excitations which form a broad peak centered at $Q=1.35 \AA^{-1}$. This broad peak is present also at $T_{N}<T<T_{S}$, however it becomes asymmetric and shifts towards a smaller value of $Q$. The asymmetry of the peak further increases in the magnetically ordered phase $\left(T<T_{N}\right)^{\frac{5}{5}}$ The value of $Q$ and its temperature evolution cannot be explained within a purely spin model, whose fundamental degrees of freedom are antiferromagnetic hexagonal spin loops with $Q=1.5 \AA^{-1}$, which is larger than the experimental $Q$. The spin dynamics, however, can be understood if one assumes that spin degrees of freedom are affected by the orbital degrees of freedom. In particular, the spatial asymmetry of the peak in the intermediate phase can be understood as the consequence of the fact that at $T<T_{S}$, vanadium octahedra are flattened and $x y$-orbital at each site is occupied. This leads to a strong antiferromagnetic exchange between vanadium spins along $x y$ direction, and, as a result, spin interactions become effectively one-dimensional. Strong onedimensional spin fluctuations give rise, via spin-orbit coupling, to fluctuations of the occupations of $x z$ or $y z$ orbitals, causing the anisotropy of the neutron peak.

There were several theoretical attempts to understand the nature of the ground state of $\mathrm{ZnV}_{2} \mathrm{O}_{4}$. However, although 
it is widely accepted that orbital degrees of freedom play an important role, no consensus is reached yet about the type of the orbital ordering $(\mathrm{OO})$ in the ground state. The first attempt to explain the physics of $\mathrm{ZnV}_{2} \mathrm{O}_{4}$ focused on the spinlattice coupling mechanism, $\frac{6}{6}$ however it didn't explain why the structural and spin order occur at different temperatures. Tsunetsugu and Motome ${ }^{7}$ later addressed this issue and related the presence of two separate phase transitions at $T_{N}$ and $T_{S}$ to the interplay between geometrical frustration and $d d \sigma$ superexchange (SE) interaction between V-ions. The ground state orbital ordering suggested in Ref. 7 consists of stacked $a b$ planes with alternating orbital occupations $(x y, x z)$ and $(x y, y z)$. Hereafter we label this orbital patterns as ROO. They also showed that this ordering of orbitals can partially remove magnetic frustration and explain experimentally observed ordered magnetic structure.

On the other hand, Tchernyshyov ${ }^{8}$ pointed out that the ground state obtained in Ref. 7 is at odds with x-ray and neutron diffraction data, because it does not possess the required $I 4_{1} /$ amd space symmetry. He argued that the spin-orbit coupling should be included into consideration. He considered a purely ionic model in which spin-orbit (SO) coupling plays the major role and determines the orbital order in the tetragonal phase. He suggested the following OO: one electron on each site occupies $x y$ orbital, while the second electron is spreaded between $x z$ and $y z$ orbitals in such a way as to minimize the spin-orbit energy at each site. Hereafter we label this orbital pattern as $\mathrm{COO}$.

Recently R. Valenti et al. 9 found in the ab-initio DFT calculations that a correct space symmetry can be actually obtained within the reasoning of Ref. 7, if one includes into consideration an additional trigonal distortion. Still, the true ground state turns out to be the same as in Ref. $\underline{8}$.

The ideas of Refs. 7 and 8 were combined in the unique framework by S. Di Matteo et al. in Ref. 10. They proposed to construct a classical ground state phase diagram by considering SE interaction and SO coupling on equal footing. They demonstrated that the SO coupling is a relevant perturbation and favors the states with unquenched orbital momentum for any value of the coupling strength. They obtained a variety of phases and found that for reasonable values of SE and SO couplings, the ground state agrees with the experimentally observed one.

In this paper we extend the analysis of Ref. 10 and study the low energy excitations in vanadium spinels. We find that the magnetic excitation spectrum strongly depends on the type of the $\mathrm{OO}$, and that it is qualitatively different for the ground states with patterns that consist of real orbitals and those with the complex linear combination of orbitals, i.e. complex orbitals. The former ground state is characterized by a quenched orbital angular momentum $(L=0)$, while the later by a unquenched $(L \neq 0)$ orbital angular momentum. $\underline{11}$ The difference in the magnetic excitation spectrum arises due to the fact that the magnetic moment of the vanadium ion is formed by both spin and orbital momentum, and fluctuations of $\mathbf{L}$ contribute to the spectrum of magnetic excitations. We argue that the measurement of magnetic excitations in neutron scattering experiments can shed light on the nature of the $\mathrm{OO}$ in the ground state.

This paper is organized as follows: in Section II we introduce the model appropriate for the description of the physical properties of $\mathrm{ZnV}_{2} \mathrm{O}_{4}$. In Section III we discuss the ground state and the magnetic excitations of the system when the orbital angular momentum is quenched. We derive linear spinwave theory of $S=1$ moments interacting on the $V^{3+}$ pyrochlore lattice. In Section IV we discuss ground state and magnetic excitations of the system when the orbital angular momentum is unquenched. We show that magnetic excitations for unquenched orbital angular momentum can be described in the framework of the magnetic excitonic model. Section V presents the conclusions. Some mathematical details are given in the Appendices A and B.

\section{THE MODEL}

The minimal model describing the low energy physics of vanadium spinel is given by

$$
H=H_{\mathrm{SE}}+H_{\mathrm{a}}+H_{\mathrm{SO}} .
$$

The first term describes nearest neighbors $(n n)$ superexchange interactions between vanadium $S=1$ spins, arising from the virtual excitations $d_{i}^{2} d_{j}^{2} \rightarrow d_{i}^{1} d_{j}^{3}$. These interactions can be written as:

$$
\begin{aligned}
H_{\mathrm{SE}}^{n n}= & -\sum_{<i j>}\left[J_{0} \mathbf{S}_{i} \cdot \mathbf{S}_{j}+J_{1}\right] O_{i j} \\
& -\sum_{<i j>} J_{2}\left[1-\mathbf{S}_{i} \cdot \mathbf{S}_{j}\right] \bar{O}_{i j}
\end{aligned}
$$

where $i$ and $j$ are nearest neighbors, $J_{0}=\eta J /[1-3 \eta]$, $J_{1}=J[1-\eta] /[1-3 \eta], J_{2}=J[1+\eta] /[1+2 \eta]$ are coupling constants, $J=t^{2} / U_{1}$ is the overall energy scale $\left(t=3 / 4 t_{d d \sigma}\right.$ and $U_{1}$ is the intra-orbital Coulomb repulsion), $\eta=J_{H} / U_{1}$ is the normalized Hund's exchange. We consider only the largest the hopping term, associated with $\sigma$-bonding 13 . Such hopping is diagonal and non-zero only if the orbitals and the plane in which hopping occurs are of the same $\alpha \beta$ type $(\alpha \beta=x z, y z, x y)$. In this case orbital contributions $O_{i j}$ and $\bar{O}_{i j}$ are expressed in terms of projectors $P_{i, \alpha \beta}$ onto the occupied orbital state $\alpha \beta$ at site $i$ and $j: O_{i j}=P_{i, \alpha \beta}\left(1-P_{j, \alpha \beta}\right)+P_{j, \alpha \beta}\left(1-P_{i, \alpha \beta}\right)$ and $\bar{O}_{i j}=P_{i, \alpha \beta} P_{j, \alpha \beta}$.

To describe the anisotropy and spin-orbit coupling term, we use the fact that, when the crystal field splitting between $t_{2 g}$ and $e_{g}$ orbitals is large, the $t_{2 g}$-electrons can be represented by an effective orbital angular momentum $L^{\prime}=1, \frac{12}{1}$ The anisotropy term (the second term of the Hamiltonian (1)), is then given by

$$
H_{\mathrm{a}}=c \sum_{i} L_{z i}^{\prime 2}
$$

where $c$ is a constant. This term describes the tetragonal distortion in the $t_{2 g}$ manifold. We notice, that here for simplicity we neglect the trigonal distortion, which is small compared to the tetragonal one. 

by

The spin-orbit coupling term ( the third term in (1) is given by

$$
H_{\mathrm{SO}}=-\lambda \sum_{i} \mathbf{L}_{i}^{\prime} \cdot \mathbf{S}_{\mathbf{i}}
$$

where $\lambda$ is SO coupling constant. Note that the true angular momentum $\mathbf{L}$ is related to an effective one as $\mathbf{L} \simeq-\mathbf{L}^{\prime}$.

The parameters of the model can be estimated from the experiments. The spectroscopy dat ${ }^{14}$ yield the Hund's exchange, $J_{H} \simeq 0.68 \mathrm{eV}$ and Coulomb intra-orbital repulsion $U_{1} \simeq 6 \mathrm{eV}$. The estimate of hopping matrix element from $\mathrm{x}$ ray photoemission spectroscopy is $t \simeq-0.35 \mathrm{eV}: \underline{\underline{13}}$, so the energy scale is $J=t^{2} / U_{1} \simeq 20.4 \mathrm{meV}$. Since the SO coupling constant is $\lambda \simeq 13 \mathrm{meV}$ (Ref. 15), we can see that the super-exchange and the spin-orbit couplings are comparable and, therefore should be treated on equal footing.

\section{REAL ORBITAL ORDER}

\section{A. Ground state}

First we discuss the ground state of the super-exchange Hamiltonian alone (Eq. 2). Without the anisotropy and spinorbit terms the ground state orbital patterns consist of only real orbitals: at each site two out of three $t_{2 g}$ orbitals $x y, x z, y z$, are occupied. This type of orbital patterns is called real orbital order, as opposed to complex orbital order, when the orbital state is formed by a complex superposition of $t_{2 g}$ orbitals in such a way, that the gain of spin orbit interaction energy is maximized.

Depending on which orbitals are occupied, one obtains two types of interacting bonds and also non-interacting bonds. Consider for example a bond in the $\alpha \beta$-plane. If there is an electron occupying $\alpha \beta$ orbital only on one site of such bond, then the bond, which we label as $b_{1}$, is weakly ferromagnetic (FM), and is described by $H_{b_{1}}=-J_{0} \mathbf{S}_{i} \cdot \mathbf{S}_{j}-J_{1}$. If both $i$ and $j$ sites of $i j$ bond are occupied by $\alpha \beta$ electrons, then the bond, which we label as $b_{2}$, is strongly antiferromagnetic (AFM). The exchange coupling is then given by $H_{b_{2}}=-J_{2}\left(1-\mathbf{S}_{i} \cdot \mathbf{S}_{j}\right)$. When neither $i$ nor $j$ site have $\alpha \beta$-orbital occupied, the bond is non-interacting.

One can easily demonstrate ${ }^{10}$ that for positive $J_{0}$ and $J_{2}$, the lowest energy configuration corresponds to the state with four ferromagnetic $b_{1}$ bonds per tetrahedron. There still exist two topologically different tetrahedral configurations with four $b_{1}$ bonds, characterized by different OO patterns. One of them, the ROO state with OO patterns proposed by Tsunetsugu $\stackrel{7}{\underline{7}}$, is compatible with experimentally observed magnetic structure - it yields AFM chains running in [110] and [1 10 ] directions (see, Fig (1). The classical energy per site in the ROO state is $E_{\mathrm{ROO}}=-2 J_{1}-2 J_{2}$. However, this state is actually not the true ground state of the super-exchange Hamiltonian (2). The other state with $4 b_{1}$ bonds (the ROO-I state in our notations), in which spins of each tetrahedron form a fully collinear up-up-up-down (uuud) state, has the ground state energy $E_{\mathrm{ROO}-\mathrm{I}}=-J_{0}-2 J_{1}-2 J_{2}$, which is lower

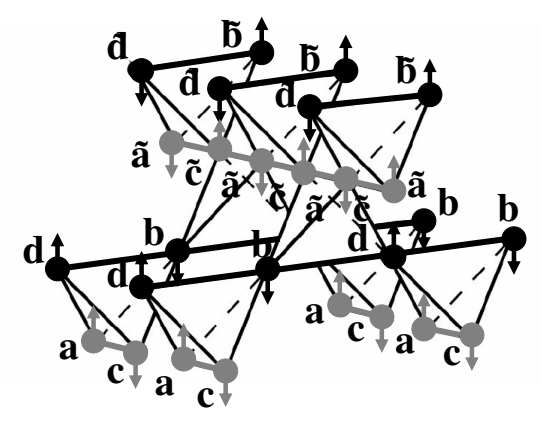

FIG. 1: (Color online) Magnetic ordering consisting of onedimensional antiferromagnetic chains in the $x y$-plane. Black and grey colors correspond to the orbital configuration in the ROO state.

than that of the ROO state. This is the lowest classical energy that one can obtain within the manifold with quenched angular momentum at each site $\frac{10}{0}$. However, the magnetic ordering associated with the ROO-I state is incompatible with the experimentally observed one: in ROO-I two neighboring spins in $x y$-plane are ferromagnetically aligned whereas the experimentally detected coupling in $x y$-plane is antiferromagnetic. This discrepancy between the ROO-I ground state of the super-exchange Hamiltonian (2) and the experimental findings demonstrates the necessity of taking into account additional interactions We study the the anisotropy term and the spin-orbit coupling in the Section IV.

One can also consider the model with both the super-exchange (SE) and the Jahn-Teller (JT) couplings ${ }^{17}$ with a hope that the cooperative JT effect, which plays an important role in the structural transition, can also stabilize the ROO type of orbital order. This model has been studied in Ref. 17 by both the mean-field analysis and Monte Carlo simulations. Here we assume that the ROO phase can be realized and in the next subsection we derive magnetic excitations spectrum for the corresponding effective spin model.

\section{B. Spin waves}

The starting point for the calculation of the magnon excitation spectrum is the classical Neel ground state with antiferromagnetic spin chains in $x y$-planes (Fig. (1). This ground state has the magnetic unit cell with eight vanadium spins, which we denote as $a, b, c, d, \tilde{a}, \tilde{b}, \tilde{c}, \tilde{d}$ (Fig 1 ). After averaging the orbital operators we can re-write the Hamiltonian (2) as

$$
H_{n n}=J_{x y} \sum_{\langle i j\rangle \| x y} \mathbf{S}_{i} \cdot \mathbf{S}_{j}+J^{\prime} \sum_{\langle i j\rangle \| x z, y z} \mathbf{S}_{i} \cdot \mathbf{S}_{j}
$$

where the first term describes the super-exchange along $x y$ chain with $J_{x y}=J_{2}$, while the second term corresponds to the frustrated ferromagnetic inter-chain coupling, $J^{\prime}=J_{0}$. To describe the excitation spectrum of such eight-sublattice antiferromagnet, we introduce eight boson operators: $a, b, c, d, \tilde{a}, \tilde{b}, \tilde{c}, \tilde{d}$. We employ Holstein-Primakoff transfor- 
mation, e.g. for up-spins $\mathbf{S}_{a}$ and down-spins $\mathbf{S}_{b}$ we have:

$$
\begin{array}{ll}
S_{a}^{z}=S-a^{\dagger} a & S_{b}^{z}=-S+b^{\dagger} b \\
S_{a}^{+}=\sqrt{2 S-a^{\dagger} a} a & S_{b}^{+}=b^{\dagger} \sqrt{2 S-b^{\dagger} b} \\
S_{a}^{-}=a^{\dagger} \sqrt{2 S-a^{\dagger} a} & S_{b}^{-}=\sqrt{2 S-b^{\dagger} b} b
\end{array}
$$

In the linear spin wave approximation we substitute $\sqrt{2 S-p^{\dagger} p}=\sqrt{2 S}$ in the expressions above. Performing Fourier transformation $p_{k}=\frac{1}{\sqrt{N}} \sum_{i} \exp ^{-\imath \vec{k} \overrightarrow{r_{i}}} p_{i}$, where $N$ is the number of lattice sites belonging to one sublattice, we obtain the mean field Hamiltonian for magnons ${ }^{16}$

$$
H=\left(\mathbf{a}^{\dagger}(\mathbf{k}),-\mathbf{a}(-\mathbf{k})\right)\left(\begin{array}{cc}
\mathbf{A}(\mathbf{k}) & \mathbf{B}(\mathbf{k}) \\
-\tilde{\mathbf{B}}(-\mathbf{k}) & -\tilde{\mathbf{A}}(-\mathbf{k})
\end{array}\right)\left(\begin{array}{c}
\mathbf{a}(\mathbf{k}) \\
\mathbf{a}^{\dagger}(-\mathbf{k})
\end{array}\right)
$$

where we introduced $\mathbf{a}(\mathbf{k})=\left(a_{\mathbf{k}}, b_{\mathbf{k}}, c_{\mathbf{k}}, d_{\mathbf{k}}, \tilde{a}_{\mathbf{k}}, \tilde{b}_{\mathbf{k}}, \tilde{c}_{\mathbf{k}}, \tilde{d}_{\mathbf{k}}\right)$. The matrices $\mathbf{A}(\mathbf{k})$ and $\mathbf{B}(\mathbf{k})$ are eight by eight matrices whose elements depend on the geometry of the lattice and the type of the magnetic ordering, tilde denotes the complex conjugation. The explicit expression for matrix elements are presented in Appendix A. The quadratic form is diagonalized using the generalized Bogoliubov transformation. In the diagonal form, the Hamiltonian takes the form:

$$
\begin{array}{r}
H_{\mathbf{k},-\mathbf{k}}=H_{\mathbf{k},-\mathbf{k}}^{0}+\sum_{n} \lambda_{n \mathbf{k}} b_{n \mathbf{k}}^{\dagger} b_{n \mathbf{k}} \\
+\sum_{n} \lambda_{n-\mathbf{k}} b_{n-\mathbf{k}}^{\dagger} b_{n-\mathbf{k}}
\end{array}
$$

where the index $n$ runs from 1 to $8, \lambda_{n \mathbf{k}}=\lambda_{n-\mathbf{k}}$ are magnon energies and $b_{n \mathbf{k}}$ are linear combinations of boson operators belonging to $\mathbf{a}(\mathbf{k})$ and $\mathbf{a}(-\mathbf{k})$.

We obtained the spin-wave excitation spectrum by solving Eq. (8) numerically. The result is presented in Fig. 2 along high-symmetry directions of 3D Brillouin zone. We used $J_{x y}=18.5 \mathrm{meV}$, and the ferromagnetic constant $J^{\prime}=$ $-0.1 J_{x y}$. There are 4 different branches of the spin-wave spectrum, each of them is doubly degenerate. Furthermore, two of the branches have zero energy over a finite range of momenta (see Fig. 2 (solid lines)). This so called zero modes emerge because the number of ferro- and antiferro-bonds connecting two neighboring antiferromagnetic chains is the same (see Fig.1), and the spins forming $x y$-chains can collectively rotate with no change in energy.

The existence of the zero modes is inconsistent with the observation of the magnetic ordering transition at $\sim 40 \mathrm{~K}$. The ordering requires that the zero mode be lifted. The issue is what interactions are responsible for the lifting of spin degeneracy.

A natural first step would be to consider longer range interactions ${ }^{17}$ as these interactions generally remove the degeneracy (it happens, e.g., in a Kagome antiferromagnet). We show below that it is indeed the case, however, the energy of the relevant degeneracy-breaking mode is very small and can not explain the magnetic ordering temperature of $\sim 40 \mathrm{~K}$.

In Fig 3 we show the interactions which include up to third neighbors. There are 6 nearest neighbor interactions $J_{n}\left(J_{n}=J_{x y}\right.$ along $x y$ chain, and $J_{n}=J^{\prime}$ along $x z$ and

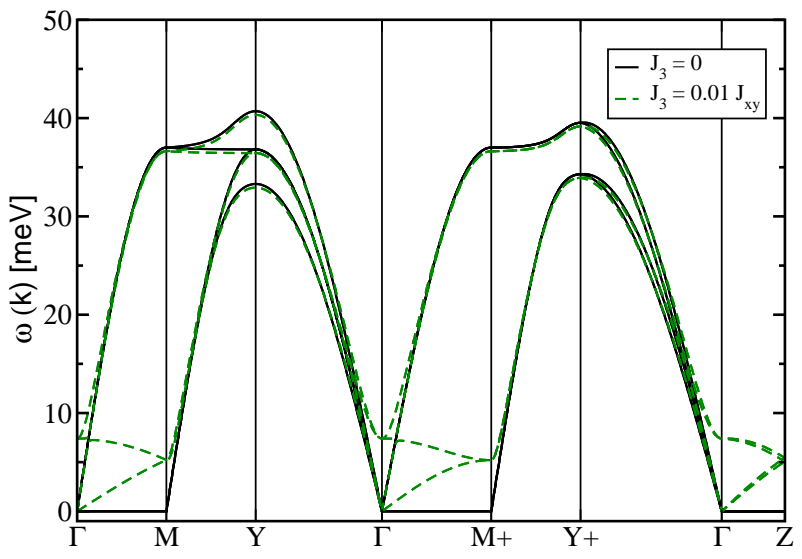

FIG. 2: (Color online) The spin-wave dispersions obtained along the main directions of the 3D BZ. Solid line corresponds to the magnon spectrum when $J_{3}=0$, dashed line - to the magnon spectrum with the correction due to the third nearest neighbor interaction with $J_{3}=$ $0.01 J_{x y}$. We have used the following labels for high-symmetry points: $\Gamma=(0,0,0), M=(\pi / 4, \pi / 4,0), Y=(0, \pi / 2,0), M+=$ $(\pi / 4, \pi / 4, \pi / 4), Y+=(0, \pi / 2, \pi / 4), Z=(0,0, \pi / 4)$.

$y z$ bonds), 12 second neighbor interactions $J_{n n}$, and 12 third neighbor interactions $J_{n n n}$. Quite often already inclusion of the second neighbor exchange lifts the degeneracy. However, here second-neighbor interactions $J_{n n}$ are frustrated and can not remove the degeneracy, and, therefore, zero modes in the spin-wave spectrum. ${ }^{17}$ Thus, one has to include third neighbor exchanges. There are two inequivalent sets of third neighbors, one obtained by two nearest neighbor steps $J_{n n n}$ and the other through the empty space $J_{n n n}^{\prime}$ (see Fig 3).

When only $d d \sigma$ hopping is considered, the exchange coupling through the empty space is zero, $J_{n n n}^{\prime}=0$, and only $J_{n n n}$ interactions contribute. These interactions are antiferromagnetic $J_{n n n}=J_{3}>0$, and non-zero only if they connect sites along the direction corresponding to the symmetry of the orbital occupation, (i.e., for orbital occupation $\alpha \beta$, the interaction is nonzero only along $\alpha \beta$ direction).

The third neighbor interaction is frustrated along $x y$-chains, but it is small compared to nearest neighbor exchange along the chain, $J_{3} \ll J_{x y}$, and can not destroy antiferromagnetic ordering along the chain. Along $x z$ and $y z$ directions, $J_{3}$ are not frustrated, and connect parallel antiferromagnetic chains located in second neighboring $x y$ planes. The energy scale for $J_{3}$ is then $\frac{t_{d d \sigma}^{4}}{U_{1}^{3}}$. This is a very small energy, only about one percent of the frustrated ferromagnetic inter-chain coupling $J^{\prime} \sim \frac{t_{d d \sigma}^{2}}{U_{1}}$. This small interaction can not explain the magnetic ordering temperature of $\sim 40 \mathrm{~K} \cdot 18$ Here we consider this interaction only qualitatively and assume the value of coupling constant $J_{3}=0.01 J_{x y}$. One can see in Fig. 2 (dashed lines) that the zero energy modes indeed become dispersive for $J_{3}>0$.

We would like to note that experimentally it has been proven that all magnetic moments are aligned along the $z$ direction. This experimental fact cannot be explained in the framework of the SE model, because it is isotropic in a spin 


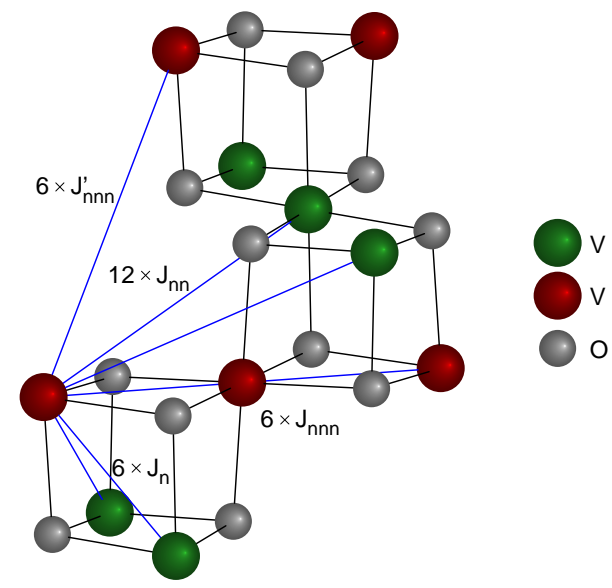

FIG. 3: (Color online) $J_{n}, J_{n n}$, and $J_{n n n}$ are first-, second- and third-neighbor exchange couplings. Second-neighbor interactions $J_{n n}$ are frustrated. The third-neighbor exchange coupling through the empty space we assume equal to zero, $J_{n n n}^{\prime}=0$; the other coupling are equal to $J_{n n n}=J_{3}$, only if they connect sites along the direction corresponding to the symmetry of orbital occupation. Red and green colors denoting vanadium ions correspond to orbital configurations in ROO state.

space. In reality, vanadium spinels likely possess a single ion spin anisotropy which aligns spin along the $z$-direction. The anisotropy does affect the magnetic excitation spectrum which in its presence acquires a gap. In this case, the zero mode will be lifted and the spin frustration will be removed. However, since the strength of magnetic anisotropy is not known experimentally at the moment, we cannot estimate the magnitude of the anisotropy-induced gap and check whether or not this interaction alone can stabilize the ground state magnetic structure. We believe that the role of the anisotropy deserves further experimental and theoretical investigation.

\section{COMPLEX ORBITAL ORDER}

\section{A. Ground state}

We now consider the ground state of the system in the presence of the SO coupling and the anisotropy term (Eqs. (3) and (4)). For any finite $\lambda$ the SO coupling prefers the orbital state with the unquenched effective orbital angular momentum $L^{\prime}=1$. In such orbital state, one electron at each site occupies $x y$ orbital due to the tetragonal distortion, while the second electron occupies the complex linear combination of $x z$ and $y z$ orbitals. The effective $\mathbf{L}^{\prime}$ should then be parallel to the spin magnetic moment in order to minimize the spin-orbit energy, i.e. a spin-up site will have $L_{z}^{\prime}=1$ while a spindown site will have $L_{z}^{\prime}=-1$. As we discussed earlier, this type of orbital ordering is a $\mathrm{COO}$ state suggested first by Tchernyshyov in Ref. 8 . Its energy $E_{C O O}=-1 / 2\left[5 J_{2}+2 J_{1}\right]-\lambda$ is lower than the energy of the ROO state for a wide range of parameters (see the phase diagram in Ref. 10).

The COO state is characterized by two strong AFM bonds per tetrahedra, and its magnetic structure consists of AFM chains in $x y$-planes with the same interaction along the chain as in the ROO state. The strengths of the inter-chain coupling $J^{\prime}$ are also practically the same for the ROO and the COO states ${ }^{10}$, although $J^{\prime}=1 / 4\left[J_{2}-2 J_{0}\right]$ is antiferromagnetic in the COO state, while it is ferromagnetic in the ROO state. In this work we assume $\left|J^{\prime}\right| / J_{x y}=0.1$ in both orbital states. In the $\mathrm{COO}$ state the preferred spin direction is fixed by anisotropy term (3) via the SO coupling to be along the $z$-axis, and, therefore, spins in $x y$-chains cannot rotate freely even for only nearest-neighbor exchange along the chains. The long range order $++\ldots$ along diagonal directions in $x z$ and $y z$ planes cannot be determined by local interactions, but for simplicity we do not include next neighbor hopping terms in this part of our calculations.

\section{B. Magnetic excitons}

We now consider magnetic excitations in the $\mathrm{COO}$ state. We follow the magnetic exciton model approach of Refs. 19, 20, which is the extension of the linear spin wave theory for systems with unquenched orbital angular moment.

We consider states with the effective total angular momentum $\mathbf{J}=\mathbf{L}^{\prime}+\mathbf{S}$. Often, energy levels with different $J$ are well separated in energy and both $J$ and its $z$-projection $J_{z}$ are good quantum numbers. However, in many transition metal oxides the strength of the spin-orbit coupling and the super-exchange interaction between localized $d$-electrons are comparable, and atomic energy levels with different values of $J$ can cross each other; in such case only $J_{z}$ acts as a good quantum number. We show below that magnetic excitations in vanadium spinels $\mathrm{AV}_{2} \mathrm{O}_{4}$ can be understood as a propagation of excitations to states with a given $J_{z}$ through the crystal.

To proceed, we rewrite the Hamiltonian (1) as a sum of a single-ion Hamiltonian $H_{1}$ and the term which describes the interaction between two different ions $\mathrm{H}_{2}$ :

$$
H=H_{1}+H_{2},
$$

where

$$
\begin{aligned}
& H_{1}=H_{\mathrm{SO}}+H_{\mathrm{a}}+\sum_{i} h_{z i} S_{z i}, \\
& H_{2}=H_{\mathrm{SE}}-\sum_{i} h_{z i} S_{z i} .
\end{aligned}
$$

The molecular field part of the exchange interaction acting on site $i$ is given by $h_{z}=\sum_{r} Z_{r} J_{r}\left\langle S_{z}\right\rangle_{r} . Z_{r}$ is the number of $r$ th neighbors, $J_{r}$ is the corresponding exchange constant, and $\left\langle S_{z}\right\rangle$ is the sublattice magnetization.

First, we diagonalize the single ion Hamiltonian, $H_{1}$, in the molecular field approximation. It is convenient to express the eigenfunction $\left|J_{z}\right\rangle$ for the states splitted by the spin-orbit interaction as linear combinations of the unperturbed eigenfunctions of $L_{z}^{\prime}$ and $S_{z}$. Then $H_{1}$ can be represented as a block $9 \times 9$ matrix in the subspace of $\left|L_{z}^{\prime}, S_{z}\right\rangle$ as follows:

$$
\begin{gathered}
H_{1}\left(J_{z}= \pm 2\right)=c-\lambda \pm h_{z} \\
H_{1}\left(J_{z}= \pm 1\right)=\left(\begin{array}{cc}
c & -\lambda \\
-\lambda & \pm h_{z}
\end{array}\right)
\end{gathered}
$$




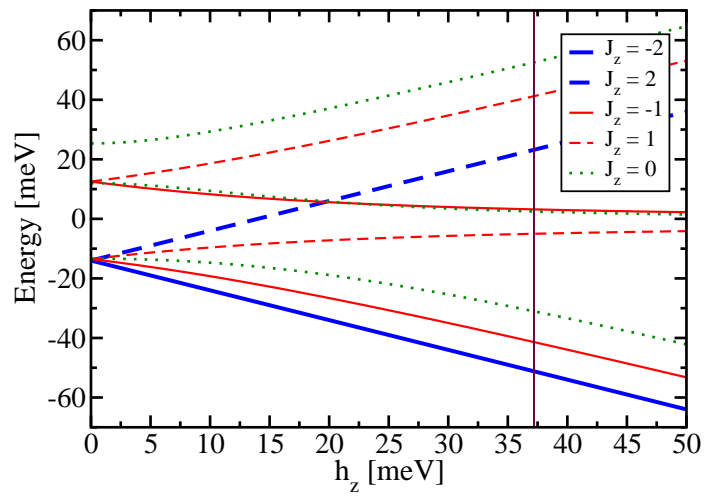

FIG. 4: (Color online) Energy levels of $\mathrm{V}^{3+}$ ions in $\mathrm{AV}_{2} \mathrm{O}_{4}$ as functions of molecular field $h_{z} . J_{z}=-2$ is a ground state for all values of $h_{z}$ (solid blue bold line). The transitions are possible only to excited states with $J_{z}=-1$ (solid red thin lines).

$$
H_{1}\left(J_{z}=0\right)=\left(\begin{array}{ccc}
c+\lambda-h_{z} & -\lambda & 0 \\
-\lambda & 0 & -\lambda \\
0 & -\lambda & c+\lambda+h_{z}
\end{array}\right)
$$

where $J_{z}=2, J_{z}=-2, J_{z}=1, J_{z}=-1$ and $J_{z}=0$ are represented in the basis $|1,1\rangle,|-1,-1\rangle,(|1,0\rangle,|0,1\rangle)$, $(|-1,0\rangle,|0,-1\rangle)$, and $(|1,-1\rangle,|0,0\rangle,|-1,1\rangle)$, respectively. Diagonalizing these matrices, we obtain eigenvalues and eigenvectors of $H_{1}$ as functions of the molecular field $h_{z}$.

When the tetrahedra are flattened as observed in the experiments, i.e. when $c<0$, for any strength of the molecular field $h_{z}$ the ground state of $H_{1}$ has $J_{z}= \pm 2$ (the sign of $J_{z}$ depends on the sign of $h_{z}$ ). For definiteness, we consider spin-down sublattice $\left(h_{z}>0\right)$. The resulting energy levels are presented in the Fig 4 . The magnetic excitations of the local Hamiltonian $H_{1}$ are transitions from the ground state to eight excited states of a single ion. These excitations can be described by boson operators $p_{\nu}^{\dagger}$ - each pseudoboson describes the transition from the ground state $|0\rangle$ to the excited state $|\nu\rangle$. The single ion Hamiltonian $H_{1}$ is diagonal in terms of pseudoboson operators:

$$
H_{1}=\sum_{i} \sum_{\nu=1}^{8} \varepsilon_{\nu} p_{\nu i}^{\dagger} p_{\nu i}
$$

where $\varepsilon_{\nu}$ is the energy difference between the excited state $|\nu\rangle$ and the ground state of $H_{1}$ :

$$
\varepsilon_{\nu}=E_{\nu}-E_{0} .
$$

We next introduce the representation for spin operators in terms of pseudobosons $p_{\nu}^{\dagger}$. The representation for $\operatorname{spin} \mathbf{S}_{p}$ on the sublattice $p$ can be written as:

$$
\begin{gathered}
S_{p}^{z}=\left\langle 0\left|S_{z}\right| 0\right\rangle+\sum_{\nu=1}^{8}\left\langle\nu\left|S_{z}\right| 0\right\rangle\left(p_{\nu}^{\dagger}+p_{\nu}\right)+ \\
\sum_{\nu=1}^{8}\left(\left\langle\nu\left|S_{z}\right| \nu\right\rangle-\left\langle 0\left|S_{z}\right| 0\right\rangle\right) p_{\nu}^{\dagger} p_{\nu} \\
S_{p}^{ \pm}=\sum_{\nu=1}^{8}\left(\left\langle\nu\left|S^{ \pm}\right| 0\right\rangle p_{\nu}^{\dagger}+\left\langle 0\left|S^{ \pm}\right| \nu\right\rangle p_{\nu}\right)
\end{gathered}
$$

If the ground state of $H_{1}$ corresponds to $J_{z}=-2$, it follows from (17) that only states with $J_{z}=-1$ contribute to $S_{p}^{ \pm}$. There are two such states, $|1\rangle$ and $|2\rangle$. In the basis of $\left|L_{z}^{\prime}, S_{z}\right\rangle$ they have the following structure:

$$
\begin{aligned}
& \left.|1\rangle=\sqrt{1-\alpha^{2}}-1,0\right\rangle+\alpha|0,-1\rangle \\
& |2\rangle=\alpha|-1,0\rangle-\sqrt{1-\alpha^{2}}|0,-1\rangle,
\end{aligned}
$$

where we denote $\alpha$ as the weight of the state with $L_{z}^{\prime}=0$, and $S_{z}=-1$ in the $|1\rangle$ state. The partial weight of orbital and spin contributions is determined by the competition between the spin-orbit coupling, the anisotropy energy and the molecular exchange field. In Fig. 5 we present a dependence of $\alpha$ on the molecular exchange field $h_{z}$ at different values of the tetragonal field $c$, keeping the SO coupling constant $\lambda$ fixed. For all values of tetragonal field parameter $\alpha$ shows similar field dependence: it increases with the increase of $h_{z}$. This happens because the molecular field $h_{z}$, acting on the spin $S_{i}$, is effectively created only by the nearest neighbors on the $x y$-chain, which are antiferromagnetically aligned to $S_{i}$. Therefore, when $h_{z}$ increases, the flipping of the spin costs more energy and the transitions with $\Delta S_{z}=1$ are suppressed. The low lying excitations become more of an orbital character, which can be seen in the increase of the weight of the transitions with $\Delta L_{z}^{\prime}=1$.

As only two excited states of an ion are relevant to the spinwave analysis, we consider the local excitations described by two pseudoboson operators $p_{1}^{\dagger}$ and $p_{2}^{\dagger}$, which take an ion from the ground state to states $|1\rangle$ and $|2\rangle$, respectively. The explicit form of extended Holstein-Primakoff transformation for spin $\mathbf{S}_{p}$ in terms $p_{1}$ and $p_{2}$ is given by:

$$
\begin{aligned}
& S_{p}^{z}=\left\langle 0\left|S_{z}\right| 0\right\rangle+ \\
&\left(\left\langle 1\left|S_{z}\right| 1\right\rangle-\left\langle 0\left|S_{z}\right| 0\right\rangle\right) p_{1}^{\dagger} p_{1}+ \\
&\left(\left\langle 2\left|S_{z}\right| 2\right\rangle-\left\langle 0\left|S_{z}\right| 0\right\rangle\right) p_{2}^{\dagger} p_{2} \\
& S_{p}^{ \pm}=<1\left|S^{ \pm}\right| 0>p_{1}^{\dagger}+<2\left|S^{ \pm}\right| 0>p_{2}^{\dagger}+ \\
&<0\left|S^{ \pm}\right| 1>p_{1}+<0\left|S^{ \pm}\right| 2>p_{2}
\end{aligned}
$$

It is also useful to rewrite these expressions, (19) and 20, using the definition of $\alpha$ (for spin-down):

$$
\begin{aligned}
S_{p}^{z} & =-1+\left(1-\alpha^{2}\right) p_{1}^{\dagger} p_{1}+\alpha^{2} p_{2}^{\dagger} p_{2} \\
S_{p}^{+} & =\sqrt{\left(1-\alpha^{2}\right) 2} p_{1}^{\dagger}+\alpha \sqrt{2} p_{2}^{\dagger} \\
S_{p}^{-} & =\sqrt{\left(1-\alpha^{2}\right) 2} p_{1}+\alpha \sqrt{2} p_{2}
\end{aligned}
$$

The interactions between localized excitations are described by $\mathrm{H}_{2}$. The excitation spectrum is obtained in a similar way as in the spin wave analysis for the ROO state. After diagonalization, the total Hamiltonian $H=H_{1}+H_{2}$ can be written in the same form as Eqs.(8), however now the index $n$ runs from 1 to 16 and the modes have complex spin-orbital character. The details of calculation are given in Appendix B.

The numerically calculated magnetic excitation spectrum for the COO state is presented in Fig 6 For comparison, we also plotted there the spin-wave dispersion for the ROO state. We used the following parameters: $J_{x y}=18.5 \mathrm{meV}$, 


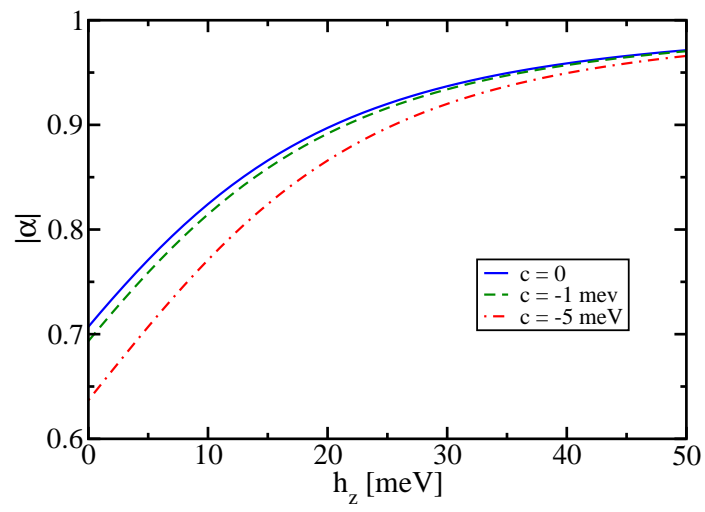

FIG. 5: (Color online) $\alpha$ as a function of molecular field $h_{z}$ for different values of the tetragonal field $c$.

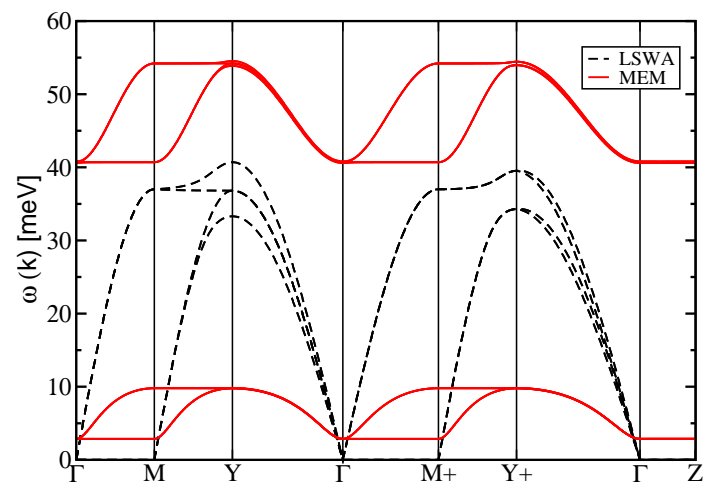

FIG. 6: Magnetic excitation spectrum. Red solid lines correspond to magnetic excitations in case of unquenched orbital angular momentum, $L \neq 0$, obtained in the framework of the magnetic exciton model (MEM). We use the following parameters: $J_{x y}=18.5 \mathrm{meV}$, $J^{\prime}=0.1 J_{x y}, c=-1 \mathrm{meV}$, and $\lambda=13 \mathrm{meV}$. The black dashed lines correspond to pure spin waves, $L=0$, obtained in the linear spin wave approximation (LSWA).The spectra are plotted along the same high symmetry directions as in Fig 2

$J^{\prime}=0.1 J_{x y}, c=-1 \mathrm{meV}$, and $\lambda=13 \mathrm{meV}$ (third neighbors interactions are not included here). As we see from Fig 6 , the excitation spectrum consists of 8 different branches, each of them is doubly degenerate. The excitations may be divided into two groups: four low lying branches with rather small gap $\Delta_{a} \sim 3 \mathrm{meV}$, and four optical branches, with the gap $\Delta_{o} \sim 35 \mathrm{meV}$. As $\Delta_{a} \ll \Delta_{o}$, the lying branches are quasiacoustic modes. As we see in Fig 6 , the lowest mode along the direction $\Gamma=(0,0,0) \rightarrow M=(\pi / 4, \pi / 4,0)$ is the dispersionless mode. This flat mode is a lifted "zero energy mode" of the ROO state. Here the zero mode is lifted due to the combined effect of the anisotropy term and the SO coupling. The tetragonal distortion favors the non-zero value of the $z$-component of orbital angular momentum, which in turn, selects the local spin quantization axis in such a way, that the $z$ component of spin is non-zero. We found that the gap, $\Delta_{a}$ is mainly determined by the anisotropy term and only weakly depends on the SO coupling (see Fig 7).

However, we note once again, that, in principle, the lifting

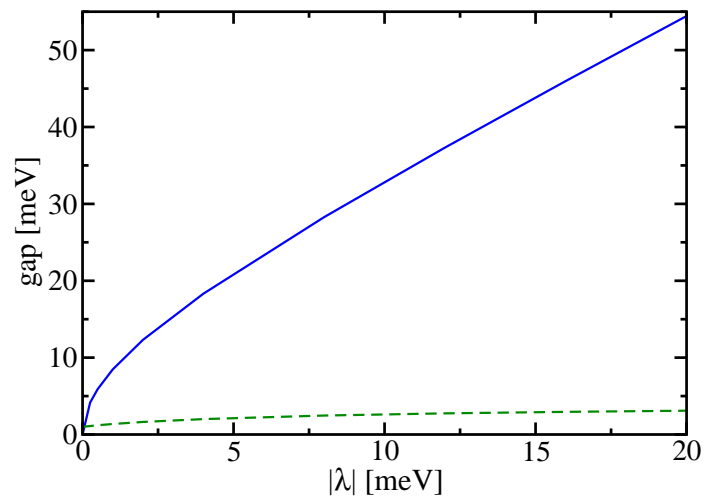

FIG. 7: (Color online) Anisotropy $\Delta_{a}$ (dashed line) and optical $\Delta_{o}$ (solid line) gaps as a function of spin-orbit coupling constant $\lambda$.

of zero energy mode can appear also in ROO state if single ion magnetic anisotropy is taken into account. We caution that the size of these two different anisotropy gaps can be of the same order, and therefore, it will be rather difficult to distinguish between them.

As a remark, we would like to mention, that such lifted zero energy modes can be detected by an inelastic neutron scattering, and have been indeed recently observed in the frustrated Kagome lattice antiferromagnet $\mathrm{KFe}_{3}(\mathrm{OH})_{6}\left(\mathrm{SO}_{4}\right)_{2}{ }^{21}$.

There is another significant difference between the magnetic excitation spectrum for $\mathrm{COO}$ and $\mathrm{ROO}$ states: as one can see from Fig 6 the bandwidth of quasi-acoustic modes in the $\mathrm{COO}$ state is strongly reduced compared to the bandwidth of pure spin waves in the ROO state. This reduction is the effect of the mixing between orbital and pure spin excitations in the spectra of the COO state. Pure orbital excitations are nondispersive, because they come form local interactions. The presence of the orbital component in the spin-wave spectrum then obviously leads to the reduction of the bandwidth.

We believe that this large reduction of the bandwidth can be seen experimentally, in the neutron scattering measurements of the magnetic excitation spectrum. Such experimental results would discriminate between two types of orbital ordering, $\mathrm{COO}$ and ROO. Unfortunately, at present these experiments are difficult to perform because to measure the full spin-wave spectrum one needs single crystals, which are still not available, as far as we know.

Let us now discuss the optical branches. These modes exist only in the COO state, and they arise from the hybridization between orbital angular momentum non-dispersive levels and dispersive spin branches due to the spin-orbit coupling. In Fig 5 we show how the optical gap, $\Delta_{o}$, depends on the strength of the spin-orbit coupling. One can see that at $\lambda=0$, $\Delta_{o}$ is zero, but it rapidly increases with increasing $\lambda$.

To summarize, the magnetic excitation spectrum for the COO state has two separated branches: quasi acoustic modes and optical modes. Both manifolds are gapped, but the gaps, $\Delta_{a}$ and $\Delta_{o}$ have different origins: $\Delta_{a}$ is set by the anisotropy term, while $\Delta_{o}$ is set by the spin-orbit coupling and is much larger.

These two gaps could be, in principle, determined by in- 
elastic neutron or Raman scattering even in powder samples of $\mathrm{ZnV}_{2} \mathrm{O}_{4}$. They are also easily distinguishable from the well-known Haldane gap, $\Delta_{H}$, which is a characteristic feature of the antiferromagnetic $S=1$ chains. $\Delta_{H}, \Delta_{a}$ and $\Delta_{o}$ have different temperature dependence: the optical gap, $\Delta_{o}$, does not depend on the temperature, as it is determined by the relativistic spin-orbit interaction; the anisotropy gap, $\Delta_{a}$, is non-zero only below the temperature of the structural transition, $T<T_{s}$; and the Haldane gap should disappear below $T_{N}$, when a long range antiferromagnetic ordering emerges.

At all temperatures, the optical gap, $\Delta_{o}$, is the largest, so we compare only the anisotropy and the Haldane gaps. At the lowest temperatures, $T<T_{N}$, the Haldane gap vanishes, and the lowest mode will have a gap equal to an anisotropy gap $\Delta_{a}$. At intermediate temperatures, $T_{N}<T<T_{S}$, the spin excitations are also gapped. In this temperature range, the gap is the sum of the anisotropy gap, $\Delta_{a}$, and the Haldane gap, $\Delta_{H}$. The magnitude of the well-developed Haldane gap is of the order of $0.4 J_{x y}$, and is compared to $T_{S}$, hence it is very likely that the gap at $T_{N}<T<T_{S}$ will be larger then at low temperatures. This behavior is exotic and, as far as we know, has not been yet observed in $S=1$ spin-chain systems. At high temperatures, $T>T_{S}$, the sharp gap is washed out by thermal fluctuations, but the spectrum can still divided into acoustic and optical branches.

\section{CONCLUSION}

We presented in this paper a detail analysis of the magnetic excitations of vanadium spinels, whose low-temperature tetragonal phase can be modeled to a high accuracy by onedimensional spin chains with weak inter-chain interaction. The formation of antiferromagnetic spin chains on the highly frustrated pyrochlore lattice is by itself non-trivial phenomena. This can happen only because vanadium ions also possess an orbital degree of freedom, and the orbital modulation of the spin exchange partially lifts the geometrical degeneracy of the underlying lattice.

We considered two different ground states: $i$ ) the one with the real orbital ordering, $\mathrm{ROO}$, and ii) the one with the complex orbital ordering, COO. We found that the excitation spectra in these two cases are qualitatively different. The spectrum for the $\mathrm{COO}$ state consists of low lying quasi-acoustic modes with small anisotropy gap and optical branches with zone-center gap determined by the spin-orbit coupling. The spectrum for the ROO state has only quasi acoustic modes. Within the superesxchange model considered in the present study the spectrum is gapless, however, in reality we expect an anisotropy gap also for this state.

The bandwidth of the quasi-acoustic modes in the COO state is strongly reduced compared to the ones for the ROO state, due to contributions from orbital $L$-modes. Because the spectra are so different, we argue that an effective way to determine experimentally the symmetry of orbital ordering in vanadium spinels is to measure their magnetic excitation spectrum.

We gratefully acknowledge discussions with P. Fulde, A.
Chubukov, S. Di Matteo, A. Loidl, H. Takagi, R. Valenti and A. Yaresko.

\section{A. Appendix A}

In this appendix we present the expressions for the matrices $\mathbf{A}(\mathbf{k})$ and $\mathbf{B}(\mathbf{k})$ in the Hamiltonian (7).

The diagonal elements of the matrix $\mathbf{A}(\mathbf{k})$ are given by

$$
\begin{array}{ll}
\mathbf{A}_{p p}(\mathbf{k})=2 J_{x y}+2 J_{3} \cos 2\left(k_{x}-k_{y}\right), & p=1,3,5,7 \\
\mathbf{A}_{p p}(\mathbf{k})=2 J_{x y}+2 J_{3} \cos 2\left(k_{x}+k_{y}\right), & p=2,4,6,8
\end{array}
$$

Nonzero matrix elements of $\mathbf{A}(\mathbf{k})$ are given by

$$
\begin{aligned}
& \mathbf{A}_{14}(\mathbf{k})=J^{\prime} e^{-i\left(k_{x}-k_{z}\right)} \\
& \mathbf{A}_{16}(\mathbf{k})=J^{\prime} e^{-i\left(k_{y}+k_{z}\right)} \\
& \mathbf{A}_{23}(\mathbf{k})=J^{\prime} e^{-i\left(k_{x}+k_{z}\right)} \\
& \mathbf{A}_{25}(\mathbf{k})=J^{\prime} e^{i\left(k_{y}+k_{z}\right)} \\
& \mathbf{A}_{38}(\mathbf{k})=J^{\prime} e^{i\left(k_{y}-k_{z}\right)} \\
& \mathbf{A}_{47}(\mathbf{k})=J^{\prime} e^{-i\left(k_{y}-k_{z}\right)} \\
& \mathbf{A}_{58}(\mathbf{k})=J^{\prime} e^{-i\left(k_{x}-k_{z}\right)} \\
& \mathbf{A}_{67}(\mathbf{k})=J^{\prime} e^{-i\left(k_{x}+k_{z}\right)}
\end{aligned}
$$

$$
\mathbf{A}_{p q}(\mathbf{k})=\tilde{\mathbf{A}}_{q p}(\mathbf{k})
$$

Nonzero matrix elements of $\mathbf{B}(\mathbf{k})$ are given by

$$
\begin{aligned}
& \mathbf{B}_{13}(\mathbf{k})=2 J_{x y} \cos \left(k_{x}-k_{y}\right) \\
& \mathbf{B}_{24}(\mathbf{k})=2 J_{x y} \cos \left(k_{x}+k_{y}\right) \\
& \mathbf{B}_{57}(\mathbf{k})=2 J_{x y} \cos \left(k_{x}-k_{y}\right) \\
& \mathbf{B}_{68}(\mathbf{k})=2 J_{x y} \cos \left(k_{x}+k_{y}\right) \\
& \mathbf{B}_{12}(\mathbf{k})=J^{\prime} e^{i\left(k_{y}+k_{z}\right)} \\
& \mathbf{B}_{18}(\mathbf{k})=J^{\prime} e^{i\left(k_{x}-k_{z}\right)} \\
& \mathbf{B}_{27}(\mathbf{k})=J^{\prime} e^{i\left(k_{x}+k_{z}\right)} \\
& \mathbf{B}_{34}(\mathbf{k})=J^{\prime} e^{-i\left(k_{y}-k_{z}\right)} \\
& \mathbf{B}_{36}(\mathbf{k})=J^{\prime} e^{-i\left(k_{x}+k_{z}\right)} \\
& \mathbf{B}_{45}(\mathbf{k})=J^{\prime} e^{-i\left(k_{x}-k_{z}\right)} \\
& \mathbf{B}_{56}(\mathbf{k})=J^{\prime} e^{i\left(k_{y}+k_{z}\right)} \\
& \mathbf{B}_{78}(\mathbf{k})=J^{\prime} e^{-i\left(k_{y}-k_{z}\right)} \\
& \mathbf{B}_{15}(\mathbf{k})=2 J_{3} \cos 2\left(k_{x}-k_{z}\right) \\
& \mathbf{B}_{26}(\mathbf{k})=2 J_{3} \cos 2\left(k_{y}-k_{z}\right) \\
& \mathbf{B}_{37}(\mathbf{k})=2 J_{3} \cos 2\left(k_{x}+k_{z}\right) \\
& \mathbf{B}_{48}(\mathbf{k})=2 J_{3} \cos 2\left(k_{y}-k_{z}\right)
\end{aligned}
$$


$\mathbf{B}_{p q}(\mathbf{k})=\tilde{\mathbf{B}}_{q p}(\mathbf{k}) \quad$ where

\section{B. Appendix B}

We next consider the modifications of the matrices for the exciton model.

In the exciton model, the base object is an enlarged set of pseudoboson operators $\hat{\mathbf{a}}(\mathbf{k})=\left(\mathbf{a}_{1}(\mathbf{k}), \mathbf{a}_{2}(\mathbf{k})\right)$, whose components $\mathbf{a}_{\mathbf{1}}(\mathbf{k})$ and $\mathbf{a}_{\mathbf{2}}(\mathbf{k})$ describe transitions to first and second excited levels. These two vectors are analogous to $\mathbf{a}(\mathbf{k})$ for pure spin wave model. As a result, the matrix $\mathbf{A}(\mathbf{k})$ is enlarged and becomes a $16 \times 16$ matrix $\hat{\mathbf{A}}(\mathbf{k})$. Its components are given by

$$
\hat{\mathbf{A}}(\mathbf{k})=\left(\begin{array}{cc}
\left(1-\alpha^{2}\right) \mathcal{A}(\mathbf{k})+\varepsilon_{1} & \alpha \sqrt{1-\alpha^{2}} \mathcal{A}(\mathbf{k}) \\
\alpha \sqrt{1-\alpha^{2}} \mathcal{A}(\mathbf{k}) & \alpha^{2} \mathcal{A}(\mathbf{k})+\varepsilon_{2}
\end{array}\right),
$$
$\hat{\mathbf{B}}(\mathbf{k})$ :
${ }^{1}$ Y. Ueda, N. Fujiwara and H.Yasuoka, J. Phys. Soc. Jpn. 66, 778 (1997).

2 S. Nizioł, Phys. Status Solidi A 18, K11 (1973).

${ }^{3}$ M. Reehuis, A. Krimmel, N. Büttgen, A. Loidl, and A. Prokofiev Eur. Phys. J. B 35, 311 (2003).

${ }^{4}$ Muhtar, F. Takagi, K. Kawakami and N. Tsuda, J. Phys. Soc. Jpn. 57, 3119 (1988).

5 S.-H. Lee et al., Phys. Rev. Lett. 93, 156407 (2004).

6 Y. Yamashita and K. Ueda, Phys. Rev. Lett. 85, 4960 (2000).

${ }^{7}$ H. Tsunetsugu, Y. Motome, Phys. Rev. B 68, 060405(R) (2003); 70, 184427 (2004).

${ }^{8}$ O. Tchernyshyov, Phys. Rev. Lett. 93, 157206 (2004).

9 T. Maitra, R. Valenti, Phys. Rev. Lett. 99, 126401 (2007).

10 S. Di Matteo, G. Jackeli, N.B. Perkins, Phys. Rev. B 72, 020408(R) (2005).

${ }^{11}$ In relation to previous works, the consideration in Ref. 7 corresponds to $L=0$, while the one in Refs. 8 and 10 corresponds to $L \neq 0$.

12 C.J. Ballhausen, Introduction to ligand field theory, McGraw-Hill, page 89 (1962).

13 K. Takubo, J.-Y. Son, T. Mizokawa, H. Ueda, M. Isobe, Y. Mat-

$$
\mathcal{A}_{p q}(\mathbf{k})= \begin{cases}\mathbf{A}_{p q}(\mathbf{k}) & p \neq q \\ 0 & p=q\end{cases}
$$

Here $p$ and $q$ runs from 1 to 8 . The diagonal matrix elements of $\mathcal{A}_{p q}(\mathbf{k})$ are zero (we consider only the case $J_{3}=0$ ), because the diagonal contribution is already included in $\varepsilon_{\nu}$.

Similarly, we can obtain the expression for the matrix

$$
\left(\begin{array}{cc}
\left(1-\alpha^{2}\right) \mathbf{B}(\mathbf{k}) & \alpha \sqrt{1-\alpha^{2}} \mathbf{B}(\mathbf{k}) \\
\alpha \sqrt{1-\alpha^{2}} \mathbf{B}(\mathbf{k}) & \alpha^{2} \mathbf{B}(\mathbf{k})
\end{array}\right)
$$

sushita, and Y. Ueda, Phys. Rev. B 74, 155103 (2006).

14 T. Mizokawa and A. Fujimori, Phys. Rev. B 54, 5368 (1996).

15 A. Abragam, and B. Bleaney, Introduction to ligand field theory, Clarendon Press - Oxford, pages 377-378 and 426-429 (1970).

${ }^{16}$ L.R. Walker, Magnetism, eds G.T. Rado and H. H. Suhl, Vol.1 (New York: Academic Press), 299-381 (1963).

17 Y. Motome and H. Tsunetsugu, Prog. Theor. Phys. Suppl. 160, 203 (2005).

18 We would like to note that the coupling between third neighbors arise not only due to the direct exchange, but also due to the various super-exchange paths through oxygen ions. In some pyrochlore compounds this contribution can be rather significant, however, here, for simplicity, we did not take this coupling into account.

19 W.J.L. Buyers, T.M. Holden, E.C. Svensson, R.A. Cowley, and M.T. Hutchings, J. Phys. C: Solid St. Phys. 4, 2139 (1971).

${ }^{20}$ K. Tomiyasu and S.Itoh, J. Phys. Soc. Jpn 75, 084708 (2006).

${ }^{21}$ K. Matan, D. Grohol, D.G. Nocera, T. Yildirim, A.B. Harris, S.H. Lee, S.E. Nagler, and Y.S. Lee, Phys. Rev. Lett. 96, 247201 (2006). 